\section{Questions et réponses} Fragen und Antworten

\section{Prévention des mycoses}

Florence Baudraz-Rosselet, Lausanne

\section{Question}

Quelles mesures de prévention (p.ex. désinfection des vêtements ou des chaussures, lessives spéciales, hygiène) doit-on proposer aux patients traités pour une onychomycose ou une mycose des pieds?

\section{Réponse}

Affections banales, fréquentes et bénignes, les mycoses des ongles et des pieds récidivent souvent. La fréquence des rechutes et des réinfections des mycoses interdigitoplantaires est difficile à évaluer car l'automédication est courante. Le taux de récidive des onychomycoses est estimé de $11-22 \%$ [1,2]. Mais dans la réalité ce chiffre pourrait être plus élevé.

Il n'est en effet pas toujours possible de différencier une réinfection d'une guérison incomplète. Certains auteurs qualifient de guérison une absence de mycose pendant 2 ans, l'examen mycologique direct étant négatif. Pour d'autres, les critères de «guérison» sont souvent inclus dans ceux d'amélioration, rendant l'évaluation globale du traitement délicate [3].

Mais tous les mycologues sont d'avis que la récidive est fréquente. La prévention doit donc être envisagée. Malheureusement, la littérature consacrée à cette question est extrêmement maigre.

Pour essayer de répondre à la question posée, plusieurs points méritent d'être éclaircis.

\section{Quels sont les facteurs favorisants de la mycose?}

Les facteurs peuvent dépendre de l'hôte et du parasite.

Parmi les facteurs dépendant de l'hôte, on relève en particulier:

- l'humidité et la macération favorisant la croissance du champignon, d'où l'atteinte élective du 4e espace inter-orteils où l'intervalle est le plus étroit, état aggravé par des chaussures trop serrées;

- une dystrophie unguéale consécutive à des traumatismes ou à des microtraumatismes répétés (chaussures mal adaptées);

- des dermatoses (p.ex. psoriasis) qui prédisposent à la colonisation d'un ongle par les dermatophytes: un ongle sain n'est en principe pas colonisable;

- le rôle du diabète dans les infections à dermatophytes qui est controversé [4];
- que les mycoses seraient plus difficiles à traiter lors d'une infection par le VIH, mais leur fréquence ne serait pas augmentée lors du SIDA [5];

- des facteurs génétiques, suggérés par Zaias et al. [6]: les dermatophyties des pieds constitueraient une affection autosomique dominante où tous les individus sont colonisés, mais dont le degré d'immunité contre le dermatophyte varie selon le patrimoine génétique.

Les facteurs dépendant de l'hôte sont donc connus et partiellement évitables par des mesures d'hygiène sur lesquelles il est toujours important d'insister.

Les facteurs liés au champignon, quant à eux, sont beaucoup moins bien connus:

- quand et sous quelle forme un champignon est-il pathogène?

- combien de temps peut-il résister dans un tissu et sous quelle forme se trouve-t-il hors de la couche cornée?

- comment les médicaments antifongiques sont-ils métabolisés par le champignon hors du substrat vivant?

La littérature ne mentionne pas de réponse univoque à ces questions. En 1982, la clinique de Zurich avait pu démontrer que le Trichophyton rubrum, le Trichophyton mentagrophytes et le Candida albicans pouvaient survivre au moins 123 jours dans l'eau chlorée de la piscine de Burgertwils et 18 jours dans celle ozonée de la Rheumaklinik [7]! Evans [communication personnelle, 1994, Boston] rapporte que la quantité de spores par mètre carré est bien supérieure en fin qu'en début de journée. Il pense également que le champignon doit être en relation avec sa squame pour être pathogène. Apparemment, hors de tout substrat organique, le champignon se trouve sous forme de résistance, de spore, et il est probablement incapable d'un quelconque métabolisme: croissance, multiplication, division...

Alors, quelle prévention proposer? Certains auteurs préconisent pour des individus à risque et lors de récidives fréquentes l'application d'une poudre antifongique [8-11]. D'autres proposent même de ne soigner que les mycoses symptomatiques, considérant que le taux de réinfections est élevé et que la prévention est peu efficace et difficilement réalisable tout en étant coûteuse.

Nous avons transmis cette question aux cliniques dermatologiques suisses et à quelques instituts étrangers, que nous remercions de leurs réponses. Certains collègues ont eu l'obligeance de rédiger des réponses particulièrement détaillées, que nous reproduisons intégralement ci-dessous.

Bibliographie

1 Hull PR: Onychomycosis - Treatment relapse and re-infection. Dermatology 1997;194(suppl):7-9.

2 Tosti A, Piraccini BM, Stinchi C, Colombo MD: Relapses of onychomycosis after successful treatment with systemic antifungals: A three-year follow up. Dermatology 1998;197:162-166.

3 Epstein E: How often does oral treatment of toenail onychomycosis produce a disease-free nail? Arch Dermatol 1998;134:15511554.

4 Lugo-Somolinos A, Sanchez JL: Prevalence of dermatophytosis in patients with diabetes. J Am Acad Dermatol 1992;26:408-410.

5 Midgley S, Hay R, Clayton Y: Atlas de poche de mycologie. Paris, Flammarion Médecine - Science, 1998, p 8.

6 Zaias N, Tosti A, Rebell G, Morelli R, Bardazzi F, Bieley H, et al: Autosomal dominant pattern of distal subungual onychomycosis caused by Trichophyton rubrum. J Am Acad Dermatol 1996;34: 302-304. 
7 Fischer E: Wie lange überleben Dermatophyten im Wasser von Hallenbädern? Dermatologica 1982;165:352-354.

8 Piérard GE, Piérard-Franchimont C: Mycoses; dans Saurat JH, Grosshans E, Laugier P, Lachapelle JM (éds): Dermatologie et maladies sexuellement transmissibles, éd 3. Paris, Masson, 1999, p 151.

9 Piérard GE, Wallace R, De Doncker P: Biometrological assessment of the preventive effect of a miconazole spray powder on athlete's foot. Clin Exp Dermatol 1996;21:344-346.

10 Feuilhade de Chauvin M, Benderdouche M, Lacroix C: Mycologie cutanée. Fiches pratiques. Novartis, février 2000, p 15.

11 Albanese G, Di Cintio R, Giorgetti P, Galbiati G, Ciampini M: Recurrent tinea pedis: A double blind study on the prophylactic use of fenticonazole powder. Mycoses 1992;35:157-159.

\section{Réponses détaillées des cliniques dermatologiques}

\section{Bâle (Prof. Dr S. Büchner)}

Bei Patienten mit Pilzinfektionen der Haut durch Dermatophyten und Candida-Spezies ist eine Dekontamination mykotisch verunreinigter Textilien mit dem im Haushalt üblichen Waschprozess durch eine $60^{\circ} \mathrm{C}$-Wäsche mit heute gebräuchlichen Vollwaschmitteln sicher gewährleistet [1]. Bei Onychound Fussmykosen wird eine vollständige Desinfektion der Strümpfe durch eine $60^{\circ} \mathrm{C}$-Maschinenwäsche mit einem modernen Vollwaschmittel erreicht.

Die Desinfektion der Schuhe bei Patienten mit Tinea pedum und Onychomykose stellt ein Problem dar [2]. Im Zweifelsfall wird der Kauf neuer Schuhe empfohlen. Als Alternative bietet sich Undecylensäure in Fungex-Puder, der in Schuhe gestreut werden kann, an.

\section{Literatur}

1 Osobski B, Tuchmann U: Der Einfluss des haushaltsüblichen Waschprozesses auf mykotisch kontaminierte Textilien. Hautarzt 1997;48:397-401.

2 Tietz HJ: Desinfektion von Schuhwerk mit Formalin noch zeitgemäss? Hautarzt 1994;45:256.

\section{Berne (Dr L. Böhlen, Prof. Dr T. Hunziker)}

Die spärliche Literatur zu diesem Thema befasst sich mit der häufigsten Lokalisation, der Fussmykose [1-3], sowie dem therapeutisch aufwendigen Nagelbefall [4]. Vorgeschlagene Massnahmen, wie z.B. Sauber- und Trockenhalten der Füsse, Tragen von Socken aus bei $60^{\circ} \mathrm{C}$ waschbarem, absorbierendem Material sowie von geeignetem, nicht traumatisierendem Schuhwerk oder Vermeiden von Barfussgehen in Nasszonen, leiten sich aus epidemiologischen Daten zu Risikosituationen ab [5], sind bezüglich ihrer Wirksamkeit aber nicht weiter belegt. Einzig zur prophylaktischen Applikation von neueren topischen Antimykotika liegen einzelne Studien vor, welche über maximal 6 Monate appliziert einen Nutzen aufzeigen [1-3]. Die modernen systemischen Antimykotika wurden in klinischen Studien bei Onychomykose bezüglich des zeitlichen Ablaufs ihrer Wirkung untersucht [6-9]: Nach Abschluss einer 3monatigen Behandlungsperiode waren nur 40-80\% der Kulturen von Nagelmaterial negativ, dieser Anteil stieg auf $80-90 \%$ nach 40-50 Wochen, um dann wieder abzusinken. Eine einzelne Studie über Tinea pedis (sog. Mokassin-Mykose) zeigte 4 Wochen nach Therapieende ebenfalls nur bei etwa $80 \%$ der behandelten Patienten eine negative Kultur des Schuppenpräparates [10].
Im Zeitalter der «evidence-based medicine» drängen sich ungelöste Fragen auf: Welches mykotisch kontaminierte Material ist in welchem Milieu wie lange infektiös und am effizientesten wie zu dekontaminieren? Welches Inokulum führt bei welchen prädisponierenden Faktoren des Patienten in der Regel zur Infektion? Ein effizienter Einsatz von präventiven Massnahmen zur Verhütung einer Reinokulation sowie einer Übertragung auf andere Individuen lässt sich erst anhand von Daten zu diesen Fragen ableiten. Gleiches gilt für die Evaluation der Aufwand-Nutzen-Relation mit der üblichen Frage nach den Kosten, ergänzt durch Fragen nach der Verträglichkeit, z.B. auch für behandelte Kleidungsstücke bzw. Schuhe oder noch wichtiger für die Umwelt. Nicht zuletzt ist bei langfristigen - bei Risikopopulationen lebenslangen? - Massnahmen die Compliance der Patienten zu berücksichtigen. Bei der spärlichen heutigen Datenlage bleiben Prozederevorschläge empirisch. Die zur Klärung notwendigen Untersuchungen erscheinen recht aufwendig und wissenschaftlich wenig spektakulär - wer wird sie an die Hand nehmen, wer wird sie finanzieren?

Beim zur Zeit unbefriedigenden Wissensstand verhalten wir uns restriktiv. Bei Behandlungsbeginn sind eine Optimierung der Körperhygiene und eine Desinfektion [11] oder allenfalls Elimination kontaminierter Kleidungsstücke oder Hygieneutensilien (z.B. Nagelschere) sowie bei Tinea pedis in den Nasszonen eine geeignete Fussbekleidung sicher sinnvoll. Im Vordergrund steht für uns jedoch die Auswahl der Patienten, welche überhaupt einer aufwendigen Therapie zugeführt werden sollen, dies insbesondere bei wenig störender Zehennagelmykose. Bei Vorliegen relevanter Risikofaktoren für einen chronischen rezidivierenden Verlauf [12] sehen wir nach eingehender Diskussion mit dem Patienten oft von einer Behandlung ab. Hierbei kommen dann zum Schutze der Mitbenutzer die angesprochenen Verhaltensmassnahmen vor allem in den Nasszonen zum Zuge. Langfristige prophylaktische Behandlungen mit topischen Antimykotika (z.B. auch Farbstoffen) sind Ausnahmefällen wie rezidivierenden bakteriellen Infekten, z.B. Erysipel bei Tinea pedis als Eintrittspforte, vorbehalten.

\section{Literatur}

1 Piérard GE, Wallace R, De Doncker P: Biometrological assessment of the preventive effect of a miconazole spray powder on athlete's foot. Clin Exp Dermatol 1996;21:344-346.

2 Albanese G, Di Cintio R, Giorgetti P, Galbiati G, Ciampini M: Recurrent tinea pedis: A double blind study on the prophylactic use of fenticonazole powder. Mycoses 1992;35:157-159.

3 Galimberti RL, Belli L, Negroni R, Castro JM, Rohwedder R, Tuculet MA: Prophylaxis of tinea pedis interdigitalis with bifonazole $1 \%$ powder. Dermatologica 1984;169(suppl):111-116.

4 Gupta AK, Daniel CR: Onychomycosis: Strategies to reduce failure and recurrence. Cutis 1998;62:189-191.

5 Weitzman I, Summerbell RC: The dermatophytes. Clin Microbiol Rev 1995;8:240-259.

6 Epstein E: How often does oral treatment of toenail onychomycosis produce a disease-free nail? Arch Dermatol 1998;134:15511554.

7 Tosti A, Piraccini BM, Stinchi C, Venturo N, Bardazzi F, Colombo MD: Treatment of dermatophyte nail infections: An open randomized study comparing intermittent terbinafine therapy with continuous terbinafine treatment and intermittent itraconazole therapy. J Am Acad Dermatol 1996;34:595-600.

8 Hofman H, Bräutigam M, Weidinger G, Zaun H, and the LAGOS II Study Group: Treatment of toenail onychomycosis. Arch Dermatol 1995;131:919-922. 


\section{Questions et réponses \\ Fragen und Antworten}

9 Heikkilä H, Stubb S: Long-term results of patients with onychomycosis treated with itraconazole. Acta Derm Venereol 1997;77:7071.

10 Gupta AK, De Doncker P, Heremans A, Stoffels P, Piérard GE, Decroix J, Heenen M, Degreef H: Itraconazole for the treatment of tinea pedis: A dosage of $400 \mathrm{mg} /$ day given for 2 to 4 weeks. J Am Acad Dermatol 1997;36:789-792.

11 Tietz H-J: Desinfektion von Schuhwerk mit Formalin noch zeitgemäss? Hautarzt 1994;45:256.

12 Daniel CR: Traditional management of onychomycosis. J Am Acad Dermatol 1996;35:S21-S25.

\section{Genève (PD Dr M. Harms)}

Mesures de prévention proposées aux patients traités pour une mycose ou onychomycose: bains

- laver à $60^{\circ} \mathrm{C}$ les chaussettes, le linge de corps, le linge de

- jeter les vieilles chaussures;

- désinfecter les chaussures en utilisant une préparation mycocide (dérivés des imidazolés), soit en spray, soit en poudre.

\section{Zurich (PD Dr R. Böni)}

Zum jetzigen Zeitpunkt und dem heutigen Stand des Wissens wird eine Formaldehyd(2-5\%)-Begasung der Schuhe empfohlen. Dabei wird Formaldehyd auf einen Watteträger gebracht und die Schuhe in einem Plastikbeutel während 2 Tagen begast. Es ist aber nach wie vor nicht sicher erwiesen, ob und in welcher Form eine Wirkung zu erwarten ist. Die Firma Janssen-Cilag bietet zu einem Unkostenbeitrag von CHF 10.- pro Schuhpaar diese Formaldehyd-Begasung von Schuhen an.

Von der Verwendung von Spezifika (antimykotische Puder, Sprays) wird aus drei Gründen abgeraten: 1. ist dieses Vorgehen relativ teuer, 2. können dadurch Resistenzen gezüchtet werden und 3. kommt es meistens nicht zu einer vollständigen Abdeckung und Erfassung der Pilze.

Die Empfehlungen für Kleider sind, diese bei $60{ }^{\circ} \mathrm{C}$ zu waschen.

\section{Conclusion}

Si nous résumons notre expérience et les avis précieux qui nous ont été transmis:

- les mesures d'hygiène sont d'actualité;

- le séchage minutieux des espaces inter-orteils est capital;

- les chaussures doivent être adaptées, tant pour limiter la macération inter-orteils que pour prévenir les microtraumatismes unguéaux;

- le lavage du linge y compris les chaussettes à $60{ }^{\circ} \mathrm{C}$ est recommandé, les spores sont détruites à cette température;

- l'application locale d'un antifongique préventif est discutable, mais apparaît, pour la plupart des auteurs, comme la mesure la plus satisfaisante [1-3];

- l'utilité de la désinfection des chaussures (voire leur élimination) n'est pas démontrée; la désinfection des chaussures au formaldéhyde est recommandée par Braun-Falco [4] (cf. rapport du Prof. Böni ci-dessus) avec mise en garde sur l'apparition possible d'un eczéma de contact (dans nos cliniques de dermatologie, la sensibilisation en Suisse est de 2\% [communication personnelle du Dr D. Perrenoud, SSDV 1998]);

- enfin, il paraît préférable d'aller tôt le matin à la piscine que tard le soir [Evans, communication personnelle, 1994, Boston]!

Remerciement

Nous remercions les Drs A.-A. Ramelet, Myriam Mock et Michel Monod ainsi que le Prof. R.G. Panizzon de leur aide précieuse dans la rédaction de ce manuscrit.

\section{Bibliographie}

1 Grigoriu D, Delacrétaz J, Borelli D: Traité de mycologie médicale. Lausanne, Payot, 1984, p 106.

2 Hay RJ, Moore M: Mycology; in Rook/Wilkinson/Ebling (eds): Textbook of Dermatology. 1998, vol 2, p 1310.

3 Martin AG, Kobayashi GS: Superficial fungal infection; in Fitzpatrick IS: Dermatology in General Medicine, éd 5. 1999, vol 2, p 2350.

4 Braun-Falco M: Dermatologie und Venerologie, éd 3. Berlin, Springer, 1984, p 203. 